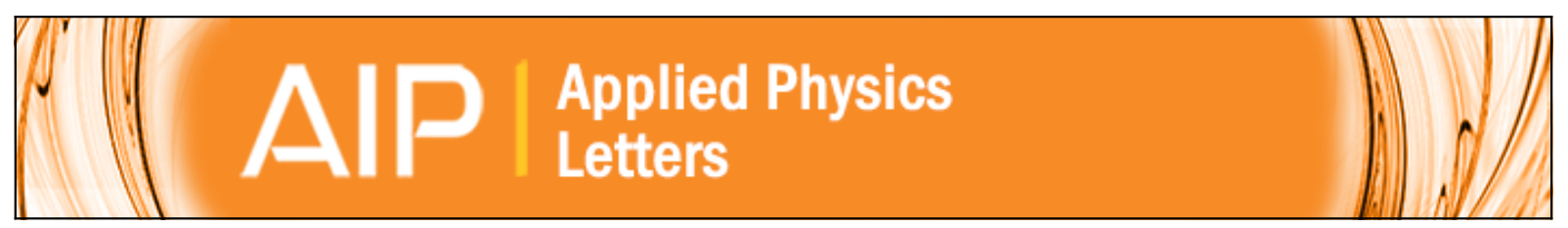

\title{
Soliton generation in active nonlinear metamaterials
}

Alexander B. Kozyrev, llya V. Shadrivov, and Yuri S. Kivshar

Citation: Applied Physics Letters 104, 084105 (2014); doi: 10.1063/1.4866856

View online: http://dx.doi.org/10.1063/1.4866856

View Table of Contents: http://scitation.aip.org/content/aip/journal/apl/104/8?ver=pdfcov

Published by the AIP Publishing

\section{Articles you may be interested in}

Low-power all-optical tunable plasmonic-mode coupling in nonlinear metamaterials

Appl. Phys. Lett. 104, 131110 (2014); 10.1063/1.4870527

Second harmonic generation from metamaterials strongly coupled to intersubband transitions in quantum wells

Appl. Phys. Lett. 104, 131104 (2014); 10.1063/1.4870072

Plasmon-induced transparency in metamaterials: Active near field coupling between bright superconducting and dark metallic mode resonators

Appl. Phys. Lett. 103, 101106 (2013); 10.1063/1.4819389

Pneumatically switchable graded index metamaterial lens

Appl. Phys. Lett. 102, 031904 (2013); 10.1063/1.4788918

Spatial resonator solitons

Chaos 13, 777 (2003); 10.1063/1.1576971

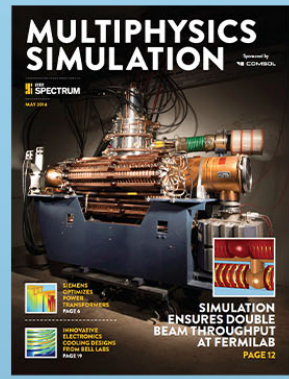

Free online magazine

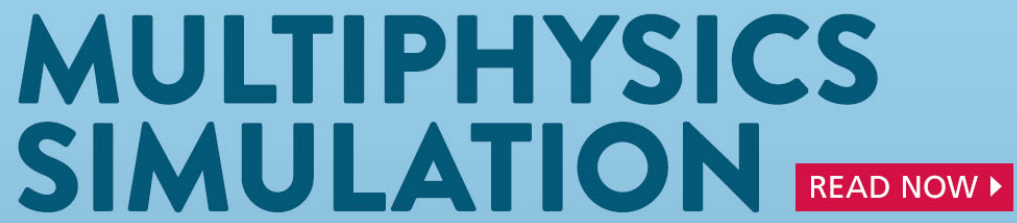




\title{
Soliton generation in active nonlinear metamaterials
}

\author{
Alexander B. Kozyrev, ${ }^{1,2}$ llya V. Shadrivov, ${ }^{1}$ and Yuri S. Kivshar ${ }^{1}$ \\ ${ }^{1}$ Nonlinear Physics Centre, Research School of Physics and Engineering, Australian National University, \\ Canberra ACT 0200, Australia \\ ${ }^{2}$ Department of Electrical and Computer Engineering, University of Wisconsin-Madison, Madison, \\ Wisconsin 53706, USA
}

(Received 6 December 2013; accepted 3 February 2014; published online 28 February 2014)

We demonstrate experimentally a mechanism of the soliton generation in nonlinear active metamaterials. Our meta-structure consists of a ring resonator formed by a microwave amplifier loaded with a left-handed transmission line. We demonstrate a variety of nonlinear effects not described by weakly nonlinear models, and study experimentally the system's spatiotemporal dynamics, including the generation of envelope solitons being realized only for the backward-wave regime. (C) 2014 AIP Publishing LLC. [http://dx.doi.org/10.1063/1.4866856]

Solitons are nonlinear pulses or beams whose spreading due to dispersion is compensated by the nonlinear self-action effect. ${ }^{1}$ In many physical systems, the soliton generation is a nontrivial process, since it requires a certain mechanism of the mode selection or filtering. In a simple case of an active cavity with a gain, the gain amplifies parasitic oscillations, and thus the process of soliton generation often results in a chaotic dynamics. The amplifier gain has to be dynamically adjusted to avoid the onset of chaos and to obtain stable periodic train of self-generated oscillations. Commonly employed methods for the soliton generation include the use of a critical threshold mechanism, frequency filters, or timegate filters with an active mode locking. These methods were employed in various physical systems such as optical ring lasers, ${ }^{1}$ fiber lasers, discrete and dissipative optical cavities, ${ }^{2-5}$ semiconductor microcavities, ${ }^{6}$ magnetic films, ${ }^{7,8}$ conventional transmission lines, ${ }^{9,10}$ and other physical systems typically described in the framework of nonlinear Schrödinger (NLS) equation or its generalizations.

In this Letter, we demonstrate experimentally the stable generation of soliton pulses in an active nonlinear metamaterial formed by a left-handed ( $\mathrm{LH})$ transmission line inserted into a resonant loop. In a sharp contrast to all previous studies, here, we employ strong and fast nonlinearity and study nonlinear dynamics in the system that stays well beyond the framework of the NLS equation. This mechanism of soliton generation does not require any external mode selection, and it can be also compared with a passive mode locking. We study the mechanism that makes soliton generation stable, and we observe self-induced periodicity, which manifests itself in the formation of spatially localized patterns within the nonlinear transmission line (NLTL).

In contrast to conventional transmission lines where nonlinearity and dispersion compensate each other to produce temporal solitons, in LH NLTL both nonlinearity and dispersion lead to waveform spreading making temporal soliton formation impossible. ${ }^{11,12}$ However, an interplay of anomalous dispersion and nonlinearity in LH NLTL may enable a variety of parametric processes, such as amplitude instability and generation of envelope solitons. ${ }^{13-15}$

For our experiments, we fabricate a LH NLTL for the set-up shown in Fig. 1. Output of the nonlinear line is connected to its input through HP 83020A wide-band power amplifier, which provides linear amplification in the transmission line band. A tunable attenuator is also connected between the output of the LH line and the amplifier (not shown) to provide a net gain control which is necessary to attain the soliton generation.

Our LH NLTL consists of 14 identical sections formed by series Skyworks Inc. SMV1233 silicon hyperabrupt varactors (two back-to-back connected diodes per section with a dc bias supply between them) and shunting Murata LQW18A00 inductors. Overall, the design of our transmission line is similar to one described in Ref. 14. In conjunction with natural parasitics, the above mentioned components form a composite right/left-handed transmission line, ${ }^{11,16,17}$ having lefthanded passband from $1.1 \mathrm{GHz}$ up to $2 \mathrm{GHz}$ for the bias voltage of interest.

It has already been demonstrated in several publications that in order to realize the scenario described by the NLS equation, the nonlinearity in LH NLTL should be very weak and the NLTL impractically long. ${ }^{15,18,19}$ In sharp contrast to the previous works, we investigate efficient soliton generation in very short transmission lines and take advantage of strong nonlinearity introduced by Schottky diodes. Even more importantly, we are taking advantage of fast nonlinearity as the nonlinear capacitance is a function of the instantaneous value of voltage along the line rather then its amplitude, a

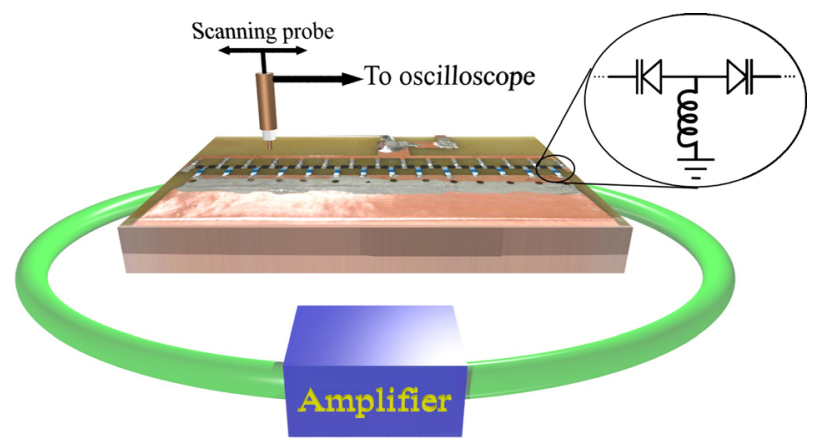

FIG. 1. Schematic experimental setup: Nonlinear left-handed transmission line output is amplified and connected to its input. Near-field probe placed above the transmission line measures the spatial distribution of the electric field in the line. 
type of nonlinearity not described in the framework of the NLS equation and its modifications developed for slow nonlinearity. Therefore, our system lies beyond the framework of NLS equation, and thus falls into a class of nonlinear systems that did not receive much attention up to date because of difficulty to realize such experimental conditions due to limited choice of nonlinearities in available materials and because of difficulty of theoretical analysis. Thus, our approach gives us a unique chance to implement and experimentally investigate strong and fast nonlinearities not commonly found in nature.

Figure 2 shows a typical waveform for the stable soliton generation measured with sampling digital wideband oscilloscope connected to the scanning probe fixed $1 \mathrm{~mm}$ above the surface and moved by a computer-controlled translation stage (see also Ref. 20). This voltage waveform is a high-frequency signal modulated so that its envelope is a periodic train of pulses. Voltage waveform propagating along the left-handed line can be written as $v(x, t)=V(x, t) \exp \{i \phi(x, t)\}$, where the function $V(x, t)$ is the slowly varying amplitude, and $\phi(x, t)$ is the phase of the local carrier signal which can be represented as $\phi(x, t)=2 \pi f t-k x-\phi_{s}(x, t)$, where $k$ and $f$ are the wavenumber and carrier frequency, respectively; $\phi_{s}(x, t)$ is an additional phase shift due to nonlinear effects.

Solid red line in Fig. 2(a) shows the function $V(x, t)$, while the phase $\phi_{s}(x, t)$ is shown by dashed line. The envelope and its phase are calculated as the amplitude and phase of the Hilbert transformation of the measured waveform. The shape of the envelope pulses resembles the cosine Jacobian elliptic function. Since the observed pulses preserve their shape from one loop to another and represent balance between dispersion and nonlinearity, as well as between gain and loss, we call these pulses dissipative solitons.

Figure 2(b) shows the spectrum of the waveform presented in Fig. 2(a). It consists of several frequency components that correspond to the phase-locked resonances of the ring structure that can be determined as follows:

$$
k_{L H}(\omega) L_{1}+k_{R H}(\omega) L_{2}+\phi=2 \pi n,
$$
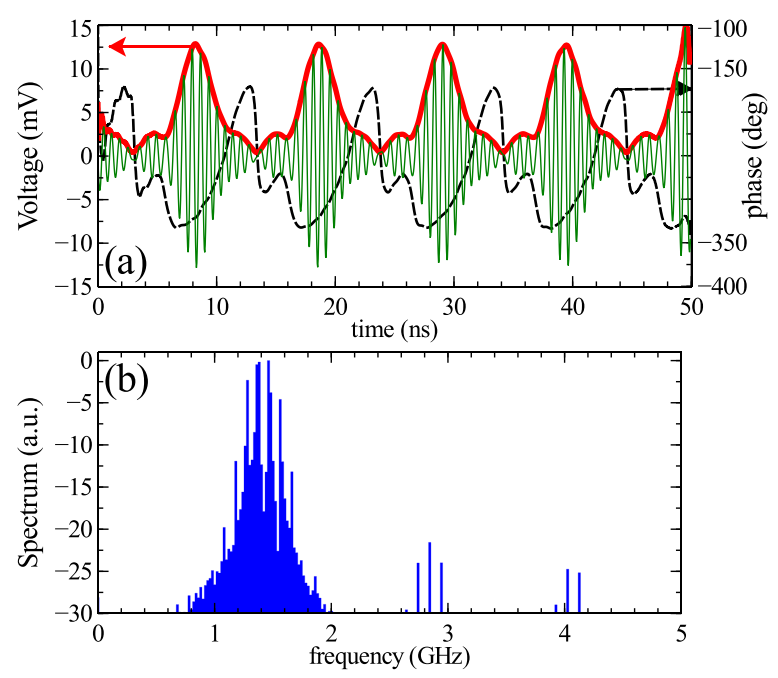

FIG. 2. Observation of solitons in left-handed transmission lines: (a) soliton waveform (solid), envelope (thick solid), and phase evolution (dashed) and (b) soliton spectrum in logarithmic scale. where $k_{L H}(\omega)$ and $L_{1}$ denote the wavenumber and the length of the left-handed transmission line, while $k_{R H}(\omega)$ and $L_{2}$ the wavenumber and length associated with connecting cables, amplifier, and tunable attenuator, and $\phi$ is the phase shift associated with the rest of the ring; $n$ is integer. We note that spectrum also shows high-harmonic generation, which is additional source of losses for the solitons. Phase shifts in the LH NLTL and in the rest of the circuit are of different sign so that phase accumulated in the NLTL is compensated by that in the linear transmission line. The frequency difference between two adjacent resonances in Eq. (1) $\Delta f$ defines the circulation period or pulse round trip time in the ring

$$
t_{r t}=\frac{1}{\Delta f}=\frac{L_{1}}{v_{g}^{L H}}+\frac{L_{2}}{v_{g}^{R H}}+t_{0},
$$

where $v_{g}^{R H}$ and $v_{g}^{L H}$ are group velocities in the right-handed and left-handed transmission line segments. Note that Eq. (2) directly follows from Eq. (1), and it is not a separate condition which should be met concurrently with Eq. (1). Circulation time defined by Eq. (2) relates to the modulation period of the measured waveforms as

$$
t_{m}=t_{r t} / m
$$

where $m$ is the number of pulses simultaneously circulating in the ring. Propagation time in the LH NLTL (measured delay for the same frequency and bias voltage) for the waveform shown in Fig. 2 is 5.6 ns. Delay in the feedback circuit $\left(L_{2} / v_{g}^{R H}\right.$ and $t_{0}$ in Eq. (2)) is $15.61 \mathrm{~ns}$, so the total circulation time is $21.21 \mathrm{~ns}$ which is twice the modulation period in Fig. 2(a). Thus, the waveform in Fig. 2(a) corresponds to $m=2$ when two pulses circulate in the ring.

In our experiments, we observe self-generation of one-, two-, and three-pulse modes, see Fig. 3. Generation of multipulse modes requires suppression of certain eigenmodes defined by Eq. (1) and amplification of others. Thus, generation of two-pulse mode requires suppression of all spectral harmonics corresponding to odd $m$ while amplifying even harmonics. This would require special experimental arrangements in employing the critical threshold mechanism which generally favors amplification of harmonics adjacent to the lowest-decay eigenmode since they usually have the next lowest decay rate (see, for example, Ref. 21). This is why it is a single pulse that is typically generated in ring resonator experiments, and why multi-soliton modes in confined system received very little attention to date. In our system, the multi-pulse mode generation is enabled without any special arrangements due to inherent mechanism of stabilization related to the interplay of anomalous dispersion and discreteness as explained below.

Figure 3 shows spatiotemporal evolution of the envelope amplitude and phase of the waveforms measured by the scanning probe. One can clearly see the pulse (pulses) circulating in the ring as distinct crests of the envelope amplitude moving with group velocity of the carrier wave (inclination of the black lines in Figs. 3(a)-3(c) corresponds to the group velocities measured with network analyzer in linear regime for the same bias voltage). The well-formed pulses suddenly appear from a cw signal or a chaotic signal in a bifurcationtype manner when the net gain and reverse bias voltage are 

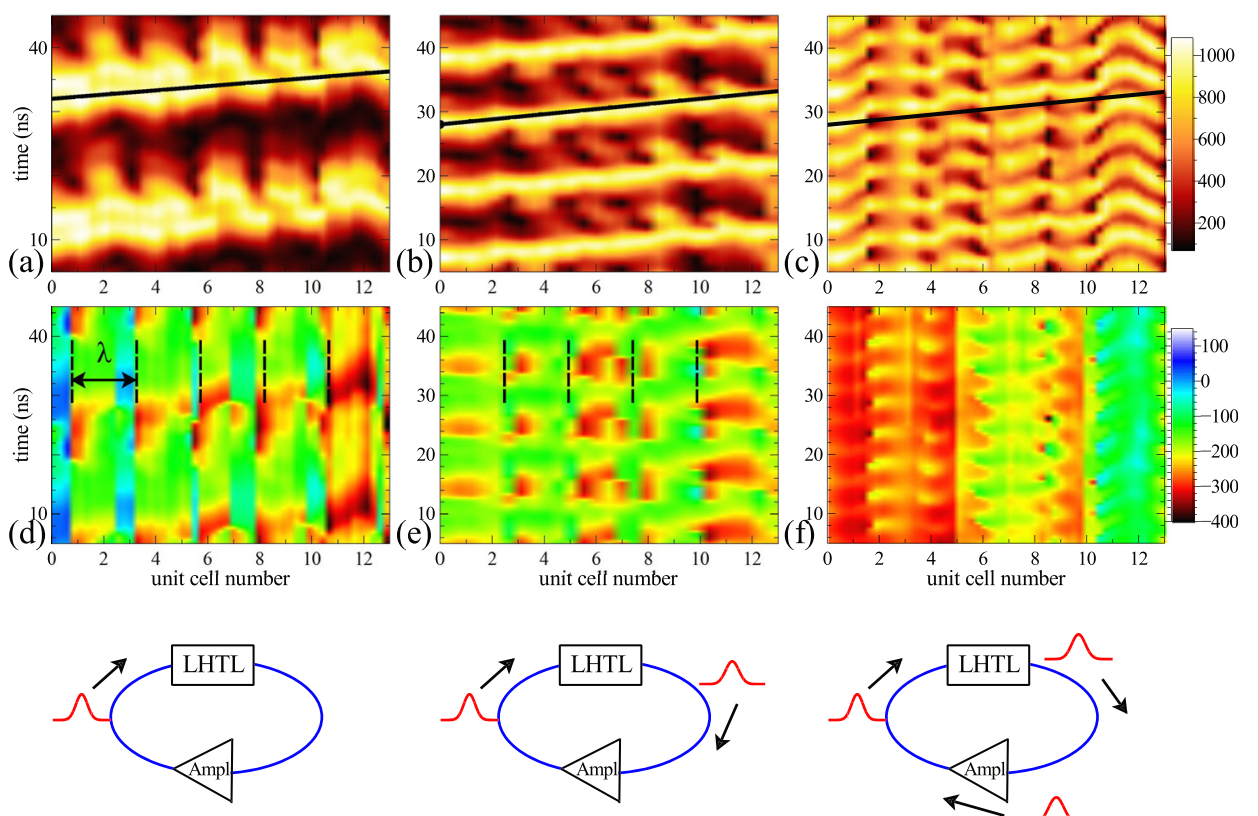

One pulse in the loop

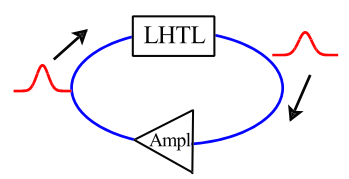

Two pulses in the loop

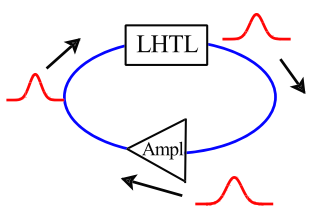

Three pulses in the loop
FIG. 3. Electric field amplitude in arbitrary units $(a, b, c)$ and phase in degrees $(\mathrm{d}, \mathrm{e}, \mathrm{f})$ dynamics in the $\mathrm{LH}$ NLTL experimentally measured at the reverse bias voltages of $11.7 \mathrm{~V}, 9.6 \mathrm{~V}$, and $13.1 \mathrm{~V}$, respectively, and shown on the plane of parameters of coordinate and time. Bottom row of plots shows schematically three regimes of soliton generation observed in the structure. varied within a certain range. The occurrence of the pulses also depends on the history of this variation thus indicating the existence of multi-stability range. Once occurred, the pulsing is stable towards small variation of bias voltage and the net gain and is accompanied by appearance of a distinct periodic field patterns (nonlinear mode build-up) overlapping with propagating pulses. They are non-moving spatially localized oscillating (or breathing) structures with distinct spatial periodicity matching the wavelength of the carrier wave. Their temporal period is equal to the circulation time of the running pulse. The periodic structure is especially well revealed in the phase distribution plots, see Figs. 3(d)-3(f) where the distance between $180^{\circ}$ phase jumps is equal to the wavelength of the carrier wave as indicated in Figs. 3(d) and 3(e) with dashed lines. It is worth mentioning that the amplitude of oscillations in the circulating and stationary modes is comparable. The net gain in the loop at the carrier frequency was $1.18 \mathrm{~dB}, 1.11 \mathrm{~dB}$, and $3.1 \mathrm{~dB}$ for one-, two-, and threepulse regimes shown in Fig. 3, while the corresponding average power measured at the input of LH NLTL was $+17 \mathrm{dBm},+15 \mathrm{dBm}$, and $+22 \mathrm{dBm}$, respectively.
Spatial periodicity does not relate to the transmission line length as we verified by also measuring the 7 unit cell transmission line. Phase shifts accumulated per one section of the transmission line are $72^{\circ}, 76^{\circ}$, and $88^{\circ}$ for regimes shown in Figs. 3(a)-3(c), respectively. Our measurements have also demonstrated that the location of the extremes of the periodic self-induced structure can be controlled by the slight variation of the bias voltage at the diodes. This allows for various pulse shapes to be generated inside and at the output of left-handed line as demonstrated in Fig. 4, which shows waveforms at different stages of transmission line for regimes shown in Figs. 3(a)-3(c).

The observed stationary periodic structure is selfinduced by propagating wave and it is a manifestation of the discrete nature of the LH NLTL. One of the important effects of the discreteness of the system is the existence of the effective periodic potential, which affects free propagation of the modes through the transmission line. ${ }^{22}$ It is connected to the fact that when moving along the chain, the pulse changes its position and, correspondingly, its structure. Thus, there are two types of modes enabled by translations of $1 / 2$ lattice
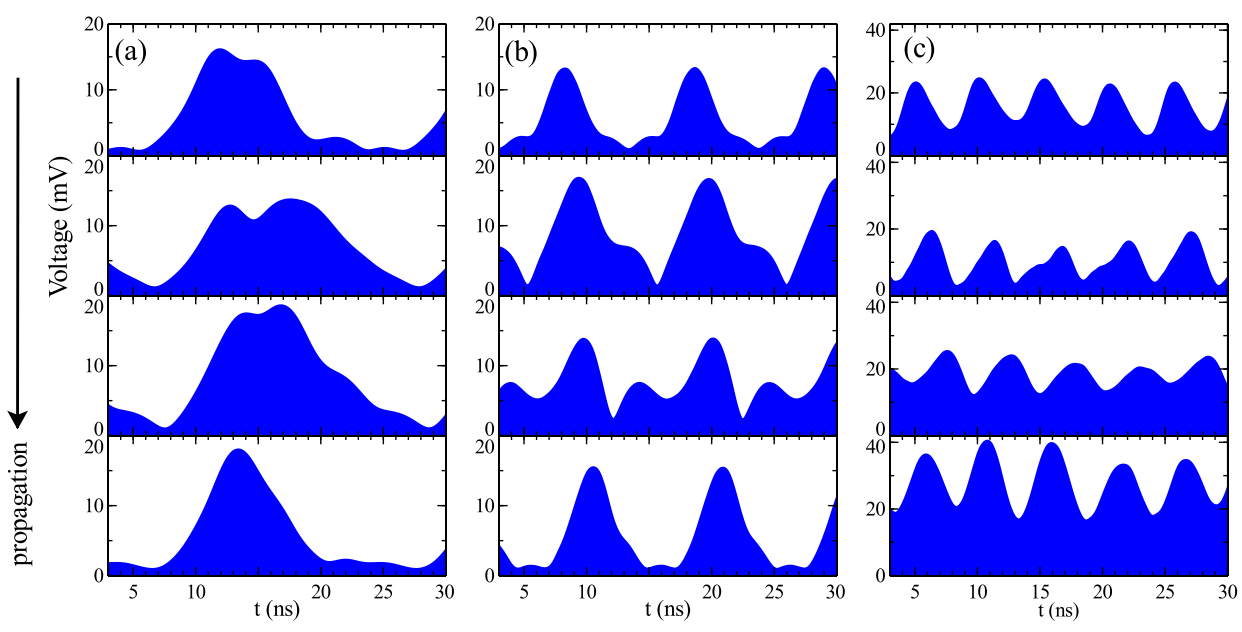

FIG. 4. Waveform envelope evolution along the LH NLTL for one (left column), two (middle column), and three (right column) soliton regimes. 
spacing and the difference in their energy may be attributed to the height of an effective periodic potential generated by the lattice discreteness. This potential resembles the PeierlsNabarro potential known in the dislocation theory. ${ }^{23}$ The existence of this potential reflects the fact that translational invariance in the system is broken by discreteness, and the translational mode no longer exists.

Due to strong nonlinearity, this self-induced periodicity of the voltage amplitude leads to a periodic variation of the capacitance along the line, resulting in a considerable change of the dispersion characteristics of the line and in enabling stop band around the carrier wave frequency. We performed a detailed investigation of the spatially localized oscillations existing in the LH structure when the circulating pulse is outside the nonlinear line and analyzed the voltage drops across the diodes. On "microscopic" level, the stationary mode corresponds to the nonlinearityinduced high-frequency branch of the splitted dispersion curve when adjacent cells oscillate out of phase. ${ }^{20}$ In contrast to the stationary oscillations, the circulating pulse corresponds to the nonlinearity-induced low-frequency branch of the dispersion curve, and the adjacent cells oscillate in phase.

Self-induced stopbands play a key role in suppressing the certain spectral harmonics. It also plays a stabilizing role preventing occurrence of stochastic instabilities due to nonlinear three- and four-wave mixing which manifest themselves in spectral line broadening. ${ }^{24}$ It allows for stable pulse generation at higher gain and higher power level (like in the three-pulse regime in our system), whereas usually higher gain in the system results in development of instabilities and stochastic generation. Thus, we have found an example of spatiotemporal localization when the temporal localization is due to ring mode-locked resonance and corresponding round-trip time in the resonant ring and the spatial localization is due to self-induced periodicity resulting from the discrete nature of the system.

In conclusion, we have demonstrated the stable generation of solitons in an active ring resonator containing only LH NLTL and an amplifier. We have observed co-existing stable self-induced patterns in the transmission line, and the generation of one, two, or three solitons in the ring. We believe our approach can be employed for other types of active negative-index metamaterials operating at $\mathrm{THz}$, infrared, and visible wavelengths. In addition, our results are of a broad interest in view of a recent agenda in the study of strong nonlinear effects with nanophotonics systems including graphene, as they may provide a hint for the use of strong external pumping for the observation of nonlinear effects, in a sharp contrast to the currently adopted strategy of loss compensation. Potential applications may include pulse forming circuits and optical comb generators for optical metrology systems.

I.V.S. and Y.S.K. acknowledge a support from the Australian Research Council.

${ }^{1}$ Yu. S. Kivshar and G. P. Agrawal, Optical Solitons: From Fibers to Photonic Crystals (Academic, San Diego, 2003), p. 530.

${ }^{2}$ M. Tlidi, P. Mandel, and R. Lefever, Phys. Rev. Lett. 73, 640 (1994).

${ }^{3}$ K. Staliunas, Phys. Rev. Lett. 91, 053901 (2003).

${ }^{4}$ O. Egorov, F. Lederer, and K. Staliunas, Opt. Lett. 32, 2106 (2007).

${ }^{5}$ M. Tlidi, P. Kockaert, and L. Gelens, Phys. Rev. A 84, 013807 (2011); P. Kockaert, P. Tassin, G. van der Sande, I. Veretennicoff, and M. Tlidi, ibid. 74, 033822 (2006).

${ }^{6}$ S. Barland, J. R. Tredicce, M. Brambilla, L. Lugiato, S. Balle, M. Giudici, T. Maggipinto, L. Spinelli, G. Tissoni, T. Knodl, M. Miller, and R. Jager, Nature 419, 699 (2002).

${ }^{7}$ B. A. Kalinikos, N. G. Kovshikov, and C. E. Patton, Phys. Rev. Lett. 80, 4301 (1998).

${ }^{8}$ B. A. Kalinikos, M. M. Scott, and C. E. Patton, Phys. Rev. Lett. 84, 4697 (2000).

${ }^{9}$ A. I. Vesnitskii, L. A. Ostrovskii, V. V. Papko, and V. N. Shabanov, Sov. Phys. JETF Lett. 9(5), 274 (1969).

${ }^{10}$ Yu. K. Bogatyrev and V. V. Papko, Radiophys. Quantum Electron. 16(7), 1035 (1973).

${ }^{11}$ A. B. Kozyrev and D. W. van der Weide, J. Phys. D: Appl. Phys. 41, 173001 (2008).

${ }^{12}$ A. B. Kozyrev, H. Kim, A. Karbassi, and D. W. van der Weide, Appl. Phys. Lett. 87, 121109 (2005).

${ }^{13}$ A. B. Kozyrev, H. Kim, and D. W. van der Weide, Appl. Phys. Lett. 88, 264101 (2006).

${ }^{14}$ A. B. Kozyrev and D. W. van der Weide, Appl. Phys. Lett. 91, 254111 (2007).

${ }^{15}$ K. Narahara, T. Nakamichi, T. Suemitsu, T. Otsuji, and E. Sano, J. Appl. Phys. 102, 024501 (2007).

${ }^{16} \mathrm{M}$. Anioniades and G. V. Eleftheriades, IEEE Antennas Wireless Propag. Lett. 2, 103 (2003).

${ }^{17}$ A. Lai, C. Caloz, and T. Itoh, IEEE Microw. Mag. 5, 34 (2004).

${ }^{18}$ G. P. Veldes, J. Cuevas, P. G. Kevrekidis, and D. J. Frantzeskakis, Phys. Rev. E 83, 046608 (2011).

${ }^{19}$ L. Q. English, S. G. Wheeler, Y. Shen, G. P. Leldes, N. Whitaker, P. G. Kevrekidis, and D. J. Frantzeskakis, Phys. Lett. A 375, 1242-1248 (2011).

${ }^{20}$ See supplementary material at http://dx.doi.org/10.1063/1.4866856 for the internal structure of the circulating pulse and the snapshots of the stationary oscillating structure.

${ }^{21}$ S. O. Demokritov, A. A. Serga, V. E. Demidov, B. Hillebrands, M. P. Kostylev, and B. A. Kalinikos, Nature 426, 159 (2003).

${ }^{22}$ Yu. S. Kivshar, W. Krolikowski, and O. A. Chubykalo, Phys. Rev. E 50, 5020 (1994).

${ }^{23}$ Yu. S. Kivshar and D. K. Campbell, Phys. Rev. E 48, 3077 (1993).

${ }^{24}$ Z. Wang, A. Hagerstrom, J. Q. Anderson, W. Tong, M. Wu, L. D. Carr, R. Eykholt, and B. A. Kalinikos, Phys. Rev. Lett. 107, 114102 (2011). 